\title{
MIGRAÇÃO PRÉ-EMPILHAMENTO EM PROFUNDIDADE NO DOMÍNIO DA FREQÜÊNCIA DE SEÇÕES DE ONDAS PLANAS
}

\author{
Celso A. Moreira Neto ${ }^{1}$, Reynam C. Pestana ${ }^{2}$ e Gary C. Aldunate ${ }^{3}$ \\ Recebido em 10 junho, 2005 / Aceito em 21 dezembro, 2005 \\ Received on June 10, 2005 / Accepted on December 21, 2005
}

\begin{abstract}
The prestack migration of common-shot section based on the wave equation has produced high quality seismic images. However, for a 3-D dataset, this process involves a huge number of computational operations and its high cost impedes its daily use in data processing. Methods that can reduce the number of migrations, as plane wave migration, requested for a complete seismic experiment, are very attractive, mainly, in the 3-D seismic data processing. Plane wave migration can also produce high quality images that are comparable to those of shot migration with much less computational cost. The prestack plane wave migration implemented in this work is applied along constant ray parameter sections, obtained through the decomposition of the data arranged in common receiver gathers taking by the SlantStack transform. Then these sections are extrapolated in depth using the Split-Step extrapolation operator. The images in depth are obtained by summing all frequencies of interest from all migrated constant ray parameter sections. The source plane wave method was applied in two synthetic seismic datasets and the results achieved with the new migration method simulating a plane wave source has produced good quality subsurface images, comparable with those from common-shot gathers migration with a lower computational cost.
\end{abstract}

Keywords: Prestack depth migration, Split-Step operator, plane wave migration.

RESUMO. A migração pré-empilhamento de seção de tiro comum baseada na equação da onda tem produzido imagens sísmicas de excelente qualidade. Entretanto, este processo exige uma alta demanda computacional, principalmente em aplicações sísmicas 3-D, e seu alto custo impede o seu uso no dia-a-dia do processamento sísmico. Assim, métodos que reduzem o número de migrações requeridas para um experimento sísmico completo, como a migração de onda planas, são bastante atrativos no processamento de dados sísmicos. Os métodos de migração de ondas planas podem produzir imagens de alta qualidade, comparável às fornecidas pela migração de tiro comum com um menor custo computacional. A migração 2-D pré-empilhamento implementada neste trabalho é realizada sobre seções de parâmetro de raio constante, obtidas através da decomposição dos dados ordenados em famílias de receptor comum, aplicando-se a transformada tau-p. Em seguida, essas seções de ondas planas são extrapoladas em profundidade usando-se operadores de extrapolação Split-Step. No processo de migração, as imagens sísmicas em profundidade são obtidas somando-se as freqüências de interesse de todas as seções de parâmetro de raio migradas. 0 método de ondas planas foi testado com sucesso em dois conjuntos de dados sísmicos sintéticos. Os resultados alcançados com este novo método de migração de ondas planas são comparáveis aos fornecidos pela migração de tiro comum e possibilitando assim a obtenção de imagens de subsuperfície de boa qualidade a um baixo custo computacional.

Palavras-chave: Migração pré-empilhamento, operador Split-Step, migração de ondas planas.

\footnotetext{
${ }^{1}$ Centro de Pesquisa em Geofísica e Geologia, Universidade Federal da Bahia, Campus Universitário da Federação, 40170-290 Salvador, BA, Brasil. E-mail: camn@cpgg.ufba.br

2 Departamento de Geofísica Nuclear, Instituto de Física, Centro de Pesquisa em Geofísica e Geologia da Universidade Federal da Bahia, Campus Universitário da Federação, 40170-290 Salvador, BA, Brasil. Tel: (71) 3203-8521; Fax: (71) 3203-8501 - E-mail: reynam@cpgg.ufba.br

${ }^{3}$ Centro de Pesquisa em Geofísica e Geologia, Universidade Federal da Bahia, Campus Universitário da Federação, 40170-290 Salvador, BA, Brasil. Tel: (71) 3203-8522; Fax: (71) 3203-8501 - E-mail: gcap@cpgg.ufba.br
} 


\section{INTRODUÇ̃̃̃o}

0 desafio de realizar exploração geofísica em áreas com alta complexidade geológica tem aumentado o interesse da indústria pelos métodos de migração em profundidade baseados em soluções da equação da onda. A migração pré-empilhamento de seção de tiro comum baseada na equação da onda tem produzido imagens de melhor qualidade do que as técnicas tipo Kirchhoff. Entretanto, este processo envolve um grande número de operações computacionais e seu alto custo impede seu uso corrente na produção. Assim, métodos que reduzem o número de migrações, requeridas para um experimento sísmico completo, são bastante atrativos.

As migrações a partir de soluções da equação da onda disponíveis na indústria são as migrações baseadas na equação de raiz quadrada dupla, a migração de tiro comum (MTC) e mais recentemente a migração de ondas planas (MOP). Ultimamente, a migração de ondas planas tem recebido um maior interesse na área de imageamento sísmico (Duquet et al., 2001; Liu et al., 2002; Zhang et al., 2003).

Os dados para a migração de ondas planas são gerados aplicando-se um atraso no tempo nos vários tiros que compõem a linha sísmica. Quando os tiros estão em linha reta a aplicação desses atrasos simula uma fonte de onda plana em uma dimensão. Ou seja, este processo de superposição linear de todos os tiros da linha sísmica consegue sintetizar uma onda plana que cobre toda a área a ser imageada. A migração de uma única onda plana permite imagear a região da estrutura iluminada pelo caminho do raio, associado com essa onda plana, conforme mostra a Figura 1. Assim, a migração de várias ondas planas se faz necessária para produzir uma imagem comparável àquela fornecida pela migração de tiro comum. Neste processo de imageamento é usado um número de migrações de ondas planas, quase sempre menor do que o número de migrações de tiro, buscando produzir resultados de qualidade comparável ao da migração de tiro comum e a menor custo computacional.

A principal motivação para o desenvolvimento e a implementação de técnicas de migração de dados sintetizados em ondas planas, a partir das seções de tiro comum, é obter melhores resultados do que os apresentados pelas técnicas de migração de ondas planas ao longo dos receptores, aplicados no domínio da freqüência e número de onda, apresentados por Santos (2000), Pestana et al. (2000), Pestana \& Stoffa (2001) e Logrado (2002).

Os métodos de migração para dados de fontes de ondas planas, usando o operador de extrapolação Split-Step (Stoffa et al., 1990), apresentados aqui, têm como objetivo obter imagens de subsuperfície de boa qualidade e, assim, facilitar a interpretação e a localização de reservatórios de hidrocarbonetos a um baixo custo computacional. A migração pré-empilhamento é realizada sobre seções de parâmetro de raio constante, obtida através da decomposição de dados ordenados em famílias de receptor comum, aplicando-se a transformada tau- $p$. Em seguida, essas seções de ondas planas são extrapoladas separadamente em profundidade. As imagens em profundidade são obtidas somandose as freqüências de interesse de todas as seções de parâmetro de raio constante migradas. Os métodos de ondas planas foram aplicados com sucesso em dois conjuntos de dados sísmicos sintéticos e os resultados alcançados com esse novo método de migração, que simula dados de fontes de ondas planas, foram comparados e apresentaram imagens de qualidade semelhantes às fornecidas pela migração de tiro comum (Aldunate \& Pestana, 2004).

\section{MIGRAÇÃO DE ONDAS PLANAS}

Os dados sísmicos de fonte pontual são decompostos em ondas planas e isso envolve aplicar um atraso linear a todos os traços de um mesmo tiro. Especificamente, se denotarmos a posição das fontes por $\left(x_{s}, 0\right)$, dos receptores por $\left(x_{r}, 0\right)$ e cada seção de receptor comum por $r\left(x_{r}, z=0 ; \omega, p\right)$ a transformação dessas seções de receptor comum em ondas planas é feita usandose a transformada de Fourier ao longo das fontes, tomando-se $k_{x}=\omega p$, ou seja:

$$
\begin{gathered}
R\left(x_{r}, z=0 ; \omega, p\right)= \\
=\int r\left(x_{r}, z=0 ; x_{s}, \omega, p\right) e^{i \omega p x_{s}} d x_{s},
\end{gathered}
$$

onde $R\left(x_{r}, z=0 ; \omega, p\right)$ é um traço do campo de onda, $\omega$ a freqüência e $p=\sin (\theta) / v$ é o parâmetro do raio, que define a direção de propagação da onda plana. E neste caso, a correspondente fonte de onda é dada por:

$$
S\left(x_{r}, z=0 ; \omega, p\right)=e^{i \omega p x_{r}} .
$$

Desde que a equação da onda é linear, a condição de imagem de tiro comum pode também ser estendida para a migração de dados de fontes de ondas planas (Jing et al., 2000). Assim, temos que a condição de imagem para a migração de ondas planas é dada pela correlação cruzada dos campos no "lag" zero, isto é:

$$
I(x, z)=\sum_{p} \sum_{\omega} \bar{S}(x, z ; \omega, p) R(x, z ; \omega, p),
$$

onde $I(x, z)$ é seção sísmica final migrada, $R(x, z ; \omega, p)$, 0 campo de ondas planas sintetizados nos receptores e 


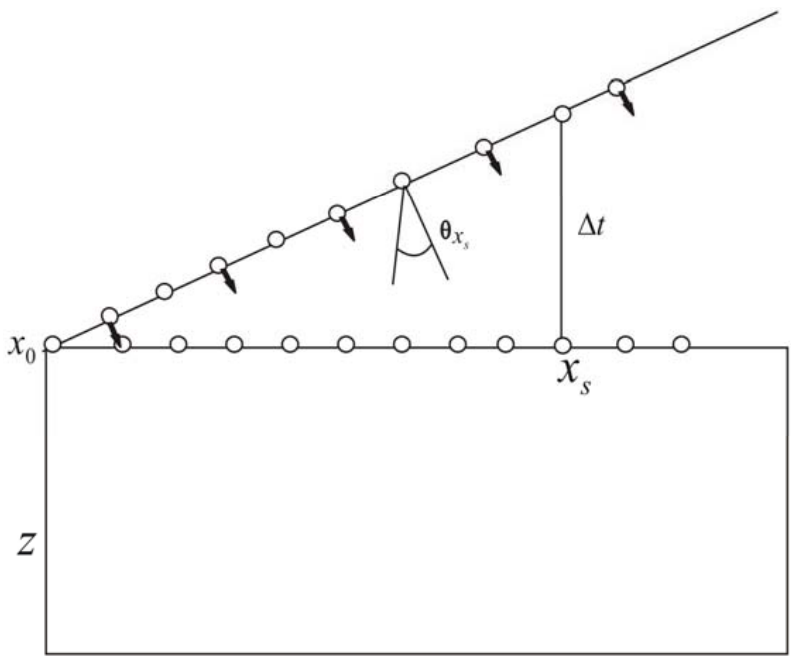

Figura 1 - Representação esquemática de uma onda plana.

$\bar{S}(x, z ; \omega, p)$ o complexo conjugado da correspondente fonte de onda plana.

Portanto, a migração de seções de ondas planas pode ser considerada um procedimento de imageamento equivalente à migração de seção de tiro comum, uma vez que a equação (3) é exatamente a condição de imagem usada na migração de tiro comum. E assim, essa técnica de migração possibilita 0 aumento da eficiência computacional das técnicas de migração de tiro comum, pois vários tiros podem ser migrados de uma única vez. Além disso, esse método de migração de ondas planas nos permite realizar a extrapolação dos campos $S(x, z ; \omega, p)$ e $R(x, z ; \omega, p)$, para a obtenção da imagem migrada, equação (3), usando-se os mesmos operadores de extrapolação usados nas migrações de tiro comum.

Assim, a extrapolação dos campos $S(x, z ; \omega, p)$ e $R(x, z ; \omega, p)$, para obtenção da imagem, equação (3), pode ser executada, por exemplo, pelo operador Split-Step apresentado por (Freire, 1988; Stoffa et al., 1990):

$$
\begin{gathered}
\tilde{P}\left(k_{x}, z+\Delta z ; \omega\right)= \\
=P\left(k_{x}, z ; \omega\right) e^{i\left[\sqrt{\left.\omega-u^{\prime}(z)\right)^{2}-k_{x}^{2}}\right] \Delta z} \\
P(x, z+\Delta z ; \omega)= \\
=\tilde{P}(x, z+\Delta z, \omega) e^{-i \omega\left[u(x, z)-u^{\prime}(z)\right] \Delta z}
\end{gathered}
$$

onde $u(x, z)$ e $u^{\prime}(z)$ são as vagarosidades verdadeira e de referência, respectivamente; $P$ é o campo de onda a ser extrapolado, com $\tilde{P}$ sendo um campo de onda intermediário.

\section{MÉTODO HíBRIDO DE MIGRAÇÃO DE ONDAS PLANAS}

Como definido pela equação (3), o processo de imageamento consiste na cross-correlação dos campos das fontes planas e dos dados sintetizados em ondas planas. Portanto, para fazer a extrapolação para baixo da seção de onda plana sintetizada, representada por $R(x, z ; \omega, p)$, que representa o resultado da combinação linear dos dados ao longo dos receptores, podemos usar 0 operador de extrapolação Split-Step (Stoffa et al., 1990). Por outro lado, se quisermos melhorar ainda a eficiência computacional deste método, podemos obter analiticamente, para os casos de modelos de velocidade simples, o tempo de percurso da onda plana, para cada ponto do modelo e assim imagear os dados sísmicos decompostos em ondas planas.

Inicialmente, consideremos o caso em que a velocidade é constante, ou seja, $v=$ const. E mais, que o modelo de velocidades seja 2-D, neste caso o campo da fonte de onda plana aplicado na superfície, $z=0$, é dada pela equação (2). Reescrevendo-a, temos que:

$$
S\left(x_{r}, z=0 ; \omega, p\right)=e^{i \omega p x_{r}} .
$$

Neste caso, temos que o campo de onda plana da fonte, em cada ponto do modelo, é dado por (Moreira, 2004):

$$
S(x, z ; \omega, p)=e^{i \omega\left(\sqrt{\frac{1}{v^{2}}-p^{2}} z+p x\right)} .
$$

Se $v=v(z)$, isto é, a velocidade varia apenas com a profundidade, temos que o campo de onda da fonte passa a ser dado agora por (Moreira, 2004):

$$
S(x, z ; \omega, p)=e^{i \omega\left(\int_{0}^{z} \sqrt{\frac{1}{v^{2}(z)}-p^{2}} d z+p x\right)} .
$$


Eassim o termo $\int_{0}^{z} \sqrt{\frac{1}{v^{2}(z)}-p^{2}} d z+p x$ é exatamente o tempo de trânsito da onda plana, com direção dada por $p$, que imerge na subsuperfície, até o ponto $(x, z)$ do modelo.

Usando o resultado dado pela equação (7), que considera apenas o tempo de trânsito da onda plana, tem-se que a condição de imagem para a migração das seções de ondas planas, passa a ser:

$$
I(x, z)=\sum_{p} \sum_{\omega} R(x, z ; \omega ; p) e^{-i \omega T(x, z, p)}
$$

$\operatorname{com} T(x, z ; p)=\int_{0}^{z} \sqrt{\frac{1}{v^{2}(z)}-p^{2}} d z+p x$.

Portanto, a migração das seções de $p$ constante torna-se mais eficiente computacionalmente, uma vez que, se aproximarmos o campo de velocidades por $v=v(z)$, podemos obter analiticamente os tempos de trânsito da fonte de ondas planas.

Para meios em que $v=v(x, z)$, isto é, com a velocidade variando tanto lateralmente como verticalmente, ainda podemos utilizar a mesma condição de imagem dada pela equação (8), porém com os tempos de trânsito da fonte de ondas planas fornecidos pela solução numérica da equação Iconal (Faria \& Stoffa, 1994).

\section{RESULTADOS NUMÉRICOS E DISCUSSÃO}

Para efeito de comparação e assim avaliar a qualidade dos resultados, bem como a eficiência computacional dos algoritmos desenvolvidos neste trabalho, utilizamos os resultados apresentados por Aldunate (2002) com o método de migração Split-Step Simultâneo (SS-S) de seções de tiro comum e também o método "Phase Shift Plus Interpolation-Split-Step" (PSPI-SS) (Aldunate \& Pestana, 2004), que é uma combinação dos métodos PSPI e SS, onde a variação lateral de velocidade é corrigida pelo método Split-Step (Stoffa et al., 1990). Neste caso, o campo de onda é extrapolado para as várias velocidades de referência e após a transformada inversa espacial de Fourier, efetua-se um segundo deslocamento de fase que visa corrigir a variação lateral de velocidade presente no campo de velocidades. Em seguida, interpolamse esses campos de onda, tal como no método PSPI, para se obter a seção sísmica migrada final.

Visando melhorar a eficácia dos métodos desenvolvidos eles são testados exaustivamente em dados sintéticos. Estes dados são mais fáceis de serem migrados, pois seus campos de velocidades são exatos e o seu completo conhecimento é fundamental para a obtenção de uma boa imagem migrada. Além do que, isto permite determinar as limitações bem como as potencialidades dos métodos desenvolvidos

Iremos utilizar dois conjuntos de dados sintéticos para tes- tar os métodos de migração pré-empilhamento 2-D no domínio das ondas planas e comparar com os resultados fornecidos pela migração de tiro comum (Aldunate, 2002) e, assim, poderemos verificar a eficiência deles em migrar estruturas complexas, bem como também identificar suas limitações em tais situações geológicas.

\section{Modelo SEG-EAGE}

Este modelo sintético foi desenvolvido em parceria entre a SEG e a EAGE. Simula uma estrutura geológica de sal com forte contraste de velocidade com o meio no qual está inserido, com vários refletores e falhas, tanto acima como abaixo do corpo de sal. Tal modelo foi baseado numa estrutura existente no Golfo do México. 0 campo de velocidades é mostrado na Figura 2, com velocidades variando de 1523 m/s a $4481 \mathrm{~m} / \mathrm{s}$, construído sobre uma malha de 645 pontos na horizontal por 150 pontos na vertical e com $24,38 \mathrm{~m}$ de espaçamento em ambas as direções. As velocidades em torno do corpo de sal são típicas dos sedimentos do Golfo do México. 0 corpo de sal atua como uma lente que dispersa 0 sinal sísmico. Neste modelo, tem-se ainda uma pequena lente localizada na posição 12000 m por 2600 m, abaixo do corpo de sal, que usaremos como um indicador, quando da sua perfeita reconstituição, que o método de migração utilizado funciona bem em tal situação geológica.

Os dados do modelo SEG-EAGE foram modelados por diferenças finitas e gerados 375 tiros com 176 receptores espaçados de 24,38 m, com um arranjo do tipo "end-on". Os dados foram amostrados a uma taxa de $8 \mathrm{~ms}$ e o tempo total de registro por seção é de $5 \mathrm{~s}$.

A seção de afastamento nulo, Figura 3, é mostrada para se ter uma idéia da complexidade da resposta sísmica, quando da presença de um corpo de sal, como descrito acima.

Para esses dados, inicialmente, realizamos a sua decomposição em ondas planas, ou melhor, em seções de parâmetros de raio ao longo dos receptores. Cada família de tiro comum foi decomposta em ondas planas e em seguida os dados ordenados em seções de $p$ constante. As seções de $p$ comum são então migradas e empilhadas para tentar reconstituir 0 modelo SEG-EAGE em profundidade (Figura 2). As imagens em profundidade, ou seja, as seções migradas foram obtidas somando-se 61 seções de parâmetros de raio de $-0,0003 \mathrm{~s} / \mathrm{m}$ a 0,0003 s/m, 0 que representa ângulos de incidência na superfície de aquisição $(z=0)$ entre $-30^{\circ} \mathrm{a}+30^{\circ}$.

A migração de dados de fontes de ondas planas com o operador Split-Step, Figura 4, conseguiu produzir um excelente resul- 


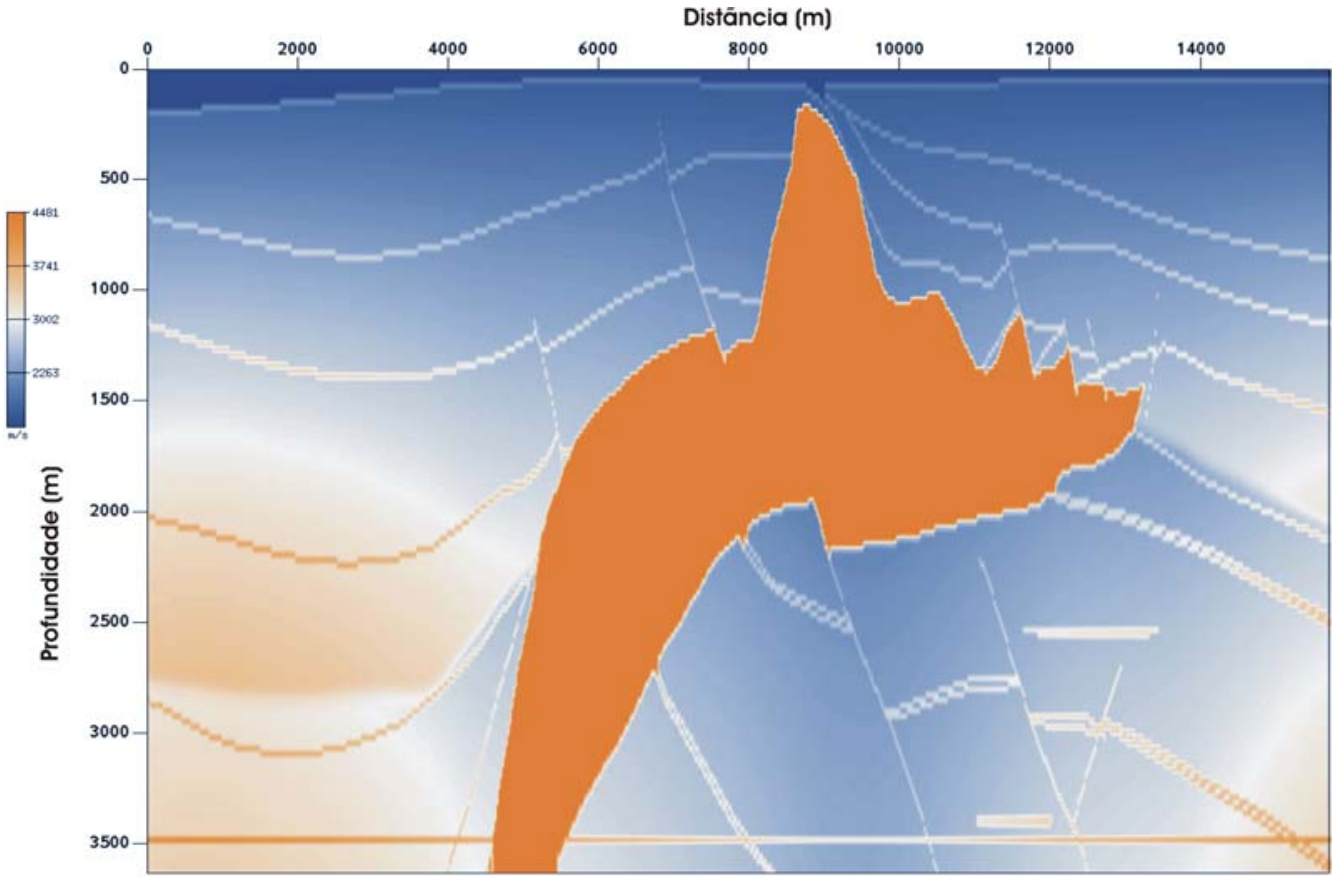

Figura 2 - Campo de velocidades do modelo SEG-EAGE simulando uma almofada de sal com vários refletores e falhamentos.

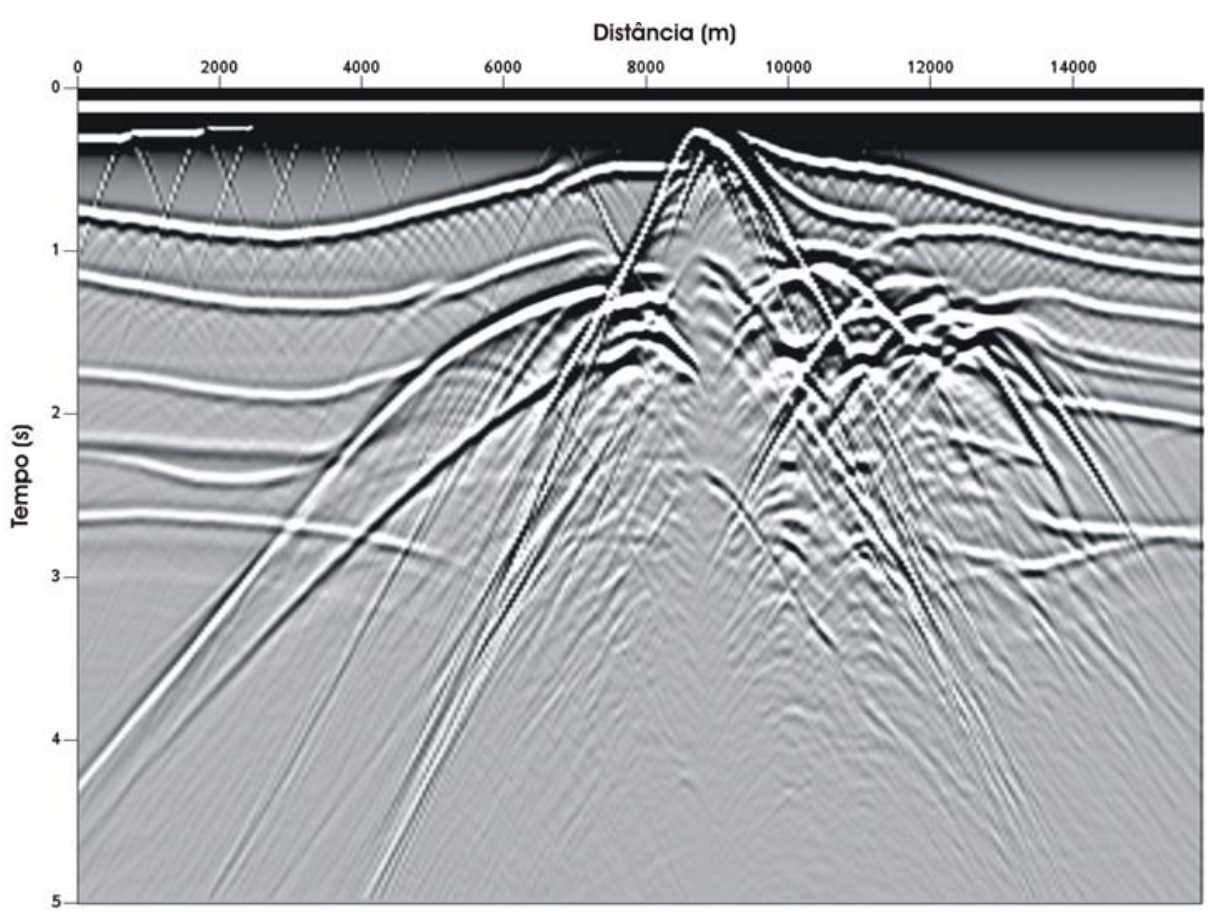

Figura 3 - Seção de afastamento nulo dos dados do modelo SEG-EAGE.

tado, ou seja, apresenta a reconstituição de todos os refletores, as falhas e os detalhes do contorno do domo de sal, principalmente na sua parte superior à direita. Este método, portanto, conse- guiu imagear satisfatoriamente os refletores abaixo do domo de sal, inclusive o flanco íngreme de difícil reconstituição, que agora aparece mais bem imageado. Também o resultado do método 


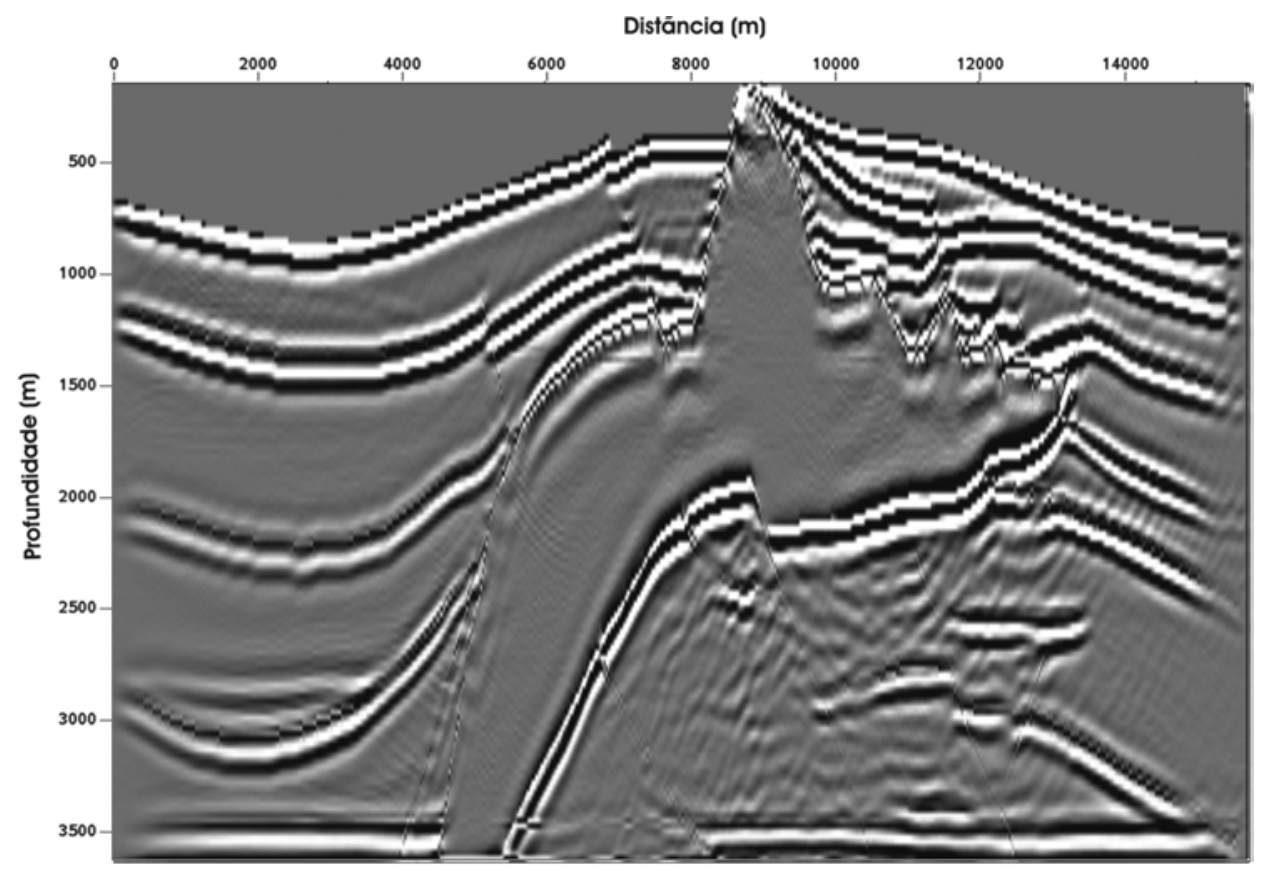

Figura 4 - Modelo SEG-EAGE após a migração de dados de fontes de ondas planas com o operador Split-Step, usando 61 seções de $p$ constante.

híbrido, Figura 5, com os tempos de percurso das ondas planas calculados a partir da solução da equação Iconal, apresenta os principais refletores desse modelo bem imageados. Por sua vez, o método híbrido foi mais rápido do que o método Split-Step anterior, porém, com um resultado inferior, principalmente na região abaixo do corpo de sal.

Do resultado da migração com o método SS-S de tiro comum, Figura 6, nota-se que este método conseguiu imagear as principais interfaces, o contorno do domo de sal e os refletores abaixo do domo. Já o resultado apresentado pelo método de fontes de ondas planas com operador Split-Step, Figura 4, é muito parecido como o método SS de tiro comum (Figura 6), porém foi conseguido a custo computacional menor como será apresentado um pouco mais à frente.

\section{Modelo SIGSBEE2A}

0 modelo SIGSBEE2A é também baseado em uma estrutura geológica real do Golfo do México, desenvolvido pelo consórcio SMAART JV e simula uma estrutura geológica complexa caracterizada por uma mudança rápida nas feições geológicas. Este modelo consiste de um corpo de sal com muitas características geométricas complexas em uma seção sedimentar de contraste de velocidade relativamente baixa (Glogovsky et al., 2002). Todas interfaces estratigráficas do modelo, como algumas falhas nor- mais do lado esquerdo e abaixo do domo de sal, que separam os blocos sedimentares são mostradas na Figura 7, entretanto as camadas estratigráficas não representam o real contraste de velocidade. Na mesma figura existem vários pontos de difração localizados a uma profundidade 5181,6 m e horizontalmente espaçados de $1524 \mathrm{~m}$. Também outra linha de difratores presente à profundidade de $7620 \mathrm{~m}$, com o mesmo espaçamento horizontal anterior.

0 campo de velocidades usado na migração está apresentado na Figura 8, onde as velocidades variam de 1499,6 m/s a 4511,0 m/s. Este campo foi construído sobre uma malha de 1066 pontos na direção horizontal, espaçados de 22,86 m, e 600 pontos em profundidade com espaçamento de 15,24 m.

Este conjunto de dados sísmicos possui 500 tiros espaçados de 45,72 m, simulando um arranjo "end-on", e cada tiro com 348 geofones espaçados de $22,86 \mathrm{~m}$. 0 tempo total de registro é de 12 segundos e 0 intervalo de amostragem usado foi de $8 \mathrm{~ms}$. Na Figura 9 mostra-se a seção de afastamento nulo, que representa a resposta sísmica deste modelo, e a partir deste podemos ter uma boa idéia da complexidade apresentada pelos dados gerados a partir do modelo SIGSBEE2A. 0 grande desafio colocado para as técnicas de migração com este conjunto de dados sísmicos é conseguir imagear o corpo de sal, que tem uma forma bastante complexa, as falhas e os refletores abaixo do domo, pois o corpo de sal não apenas atenua como também dispersa o sinal sísmico, assim como os pontos de difração. 


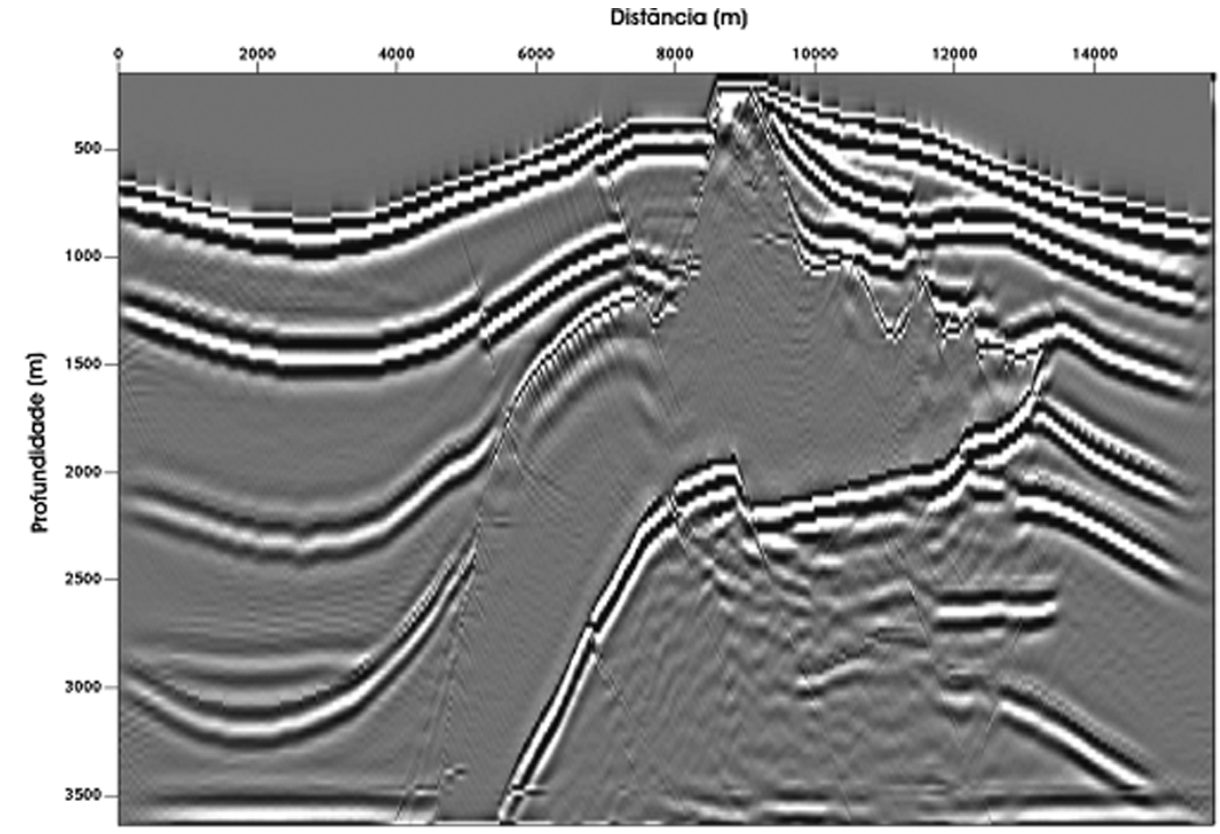

Figura 5 - Modelo SEG-EAGE após a migração de dados de fontes de ondas planas: método híbrido com 61 seções de $p$ constante.

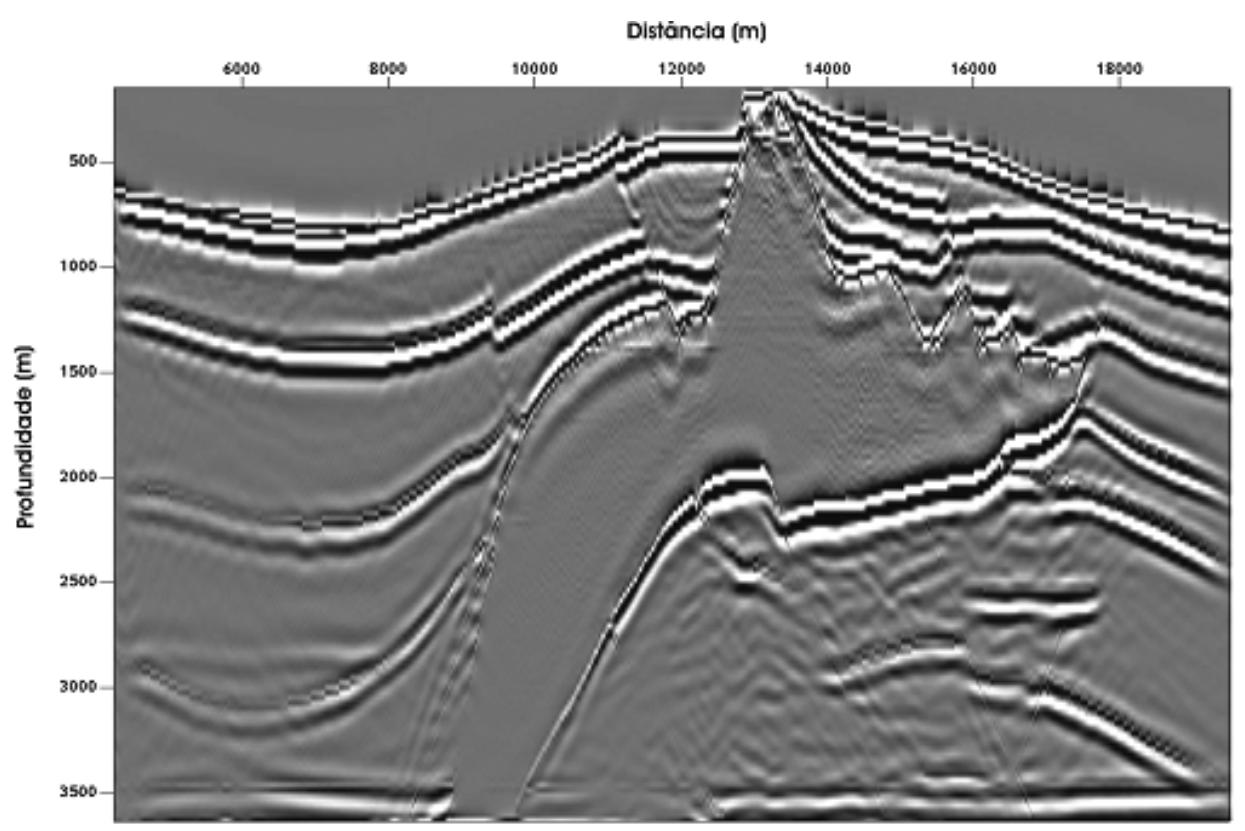

Figura 6 - Modelo SEG-EAGE após a migração SS-S de família de tiro comum.

De forma semelhante ao dado anterior, para a aplicação dos métodos de migração de dados de fontes de ondas planas com operador Split-Step e o método híbrido, os dados foram inicialmente decompostos em ondas planas. A seguir foram selecionadas as seções de parâmetro de raio constante e então migra- das. Os parâmetros de raio utilizados variam de $-0,0004 \mathrm{~s} / \mathrm{m}$ a $0,00041 \mathrm{~s} / \mathrm{m}$, que correspondem à faixa de ângulos de incidência na superfície de $-30^{\circ} \mathrm{a}+30^{\circ}$. A seção migrada final em profundidade foi gerada somando-se 81 seções de $p$ constante.

A migração dos dados de fontes de ondas planas com o ope- 


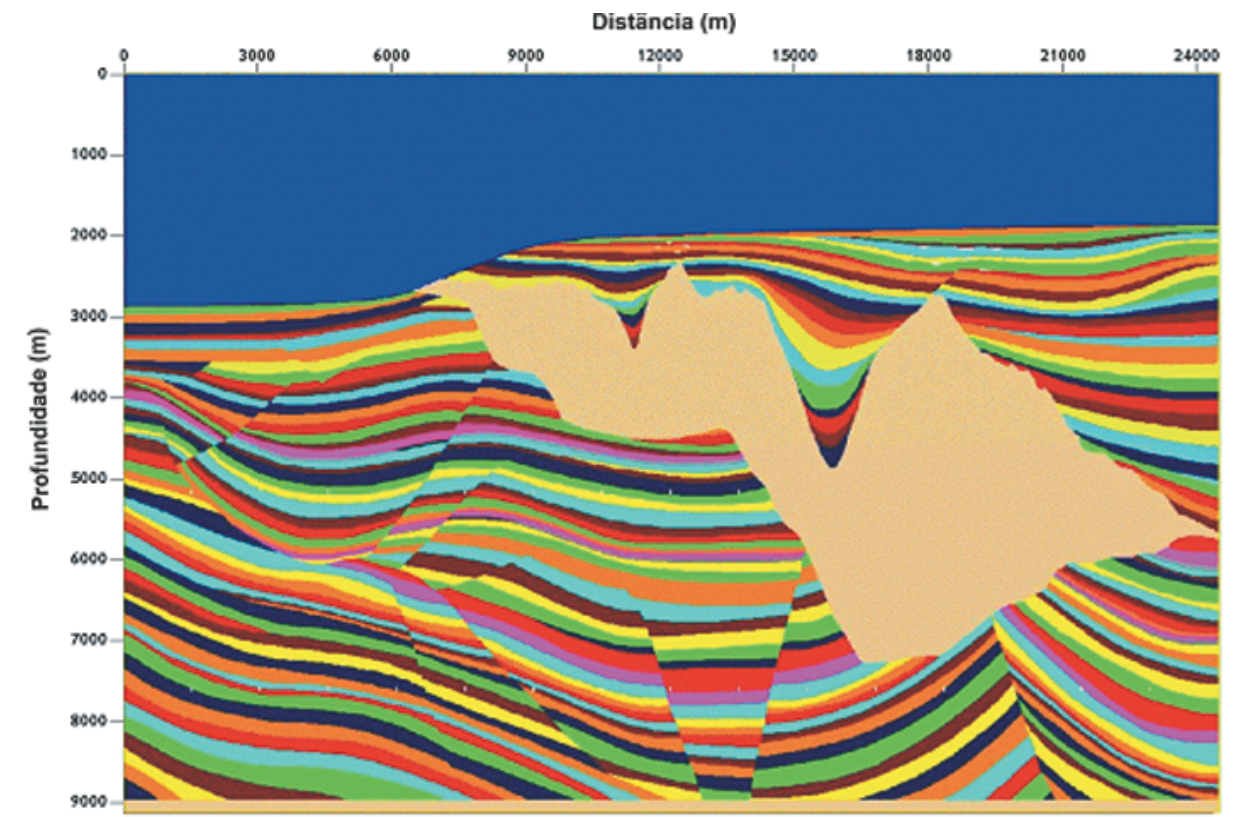

Figura 7 - Estratigrafia do modelo SIGSBEE2A: A estratigrafia das camadas mostra a disposição dos refletores, das falhas e dos pontos de difração, mas elas não representam os contrastes de velocidades.

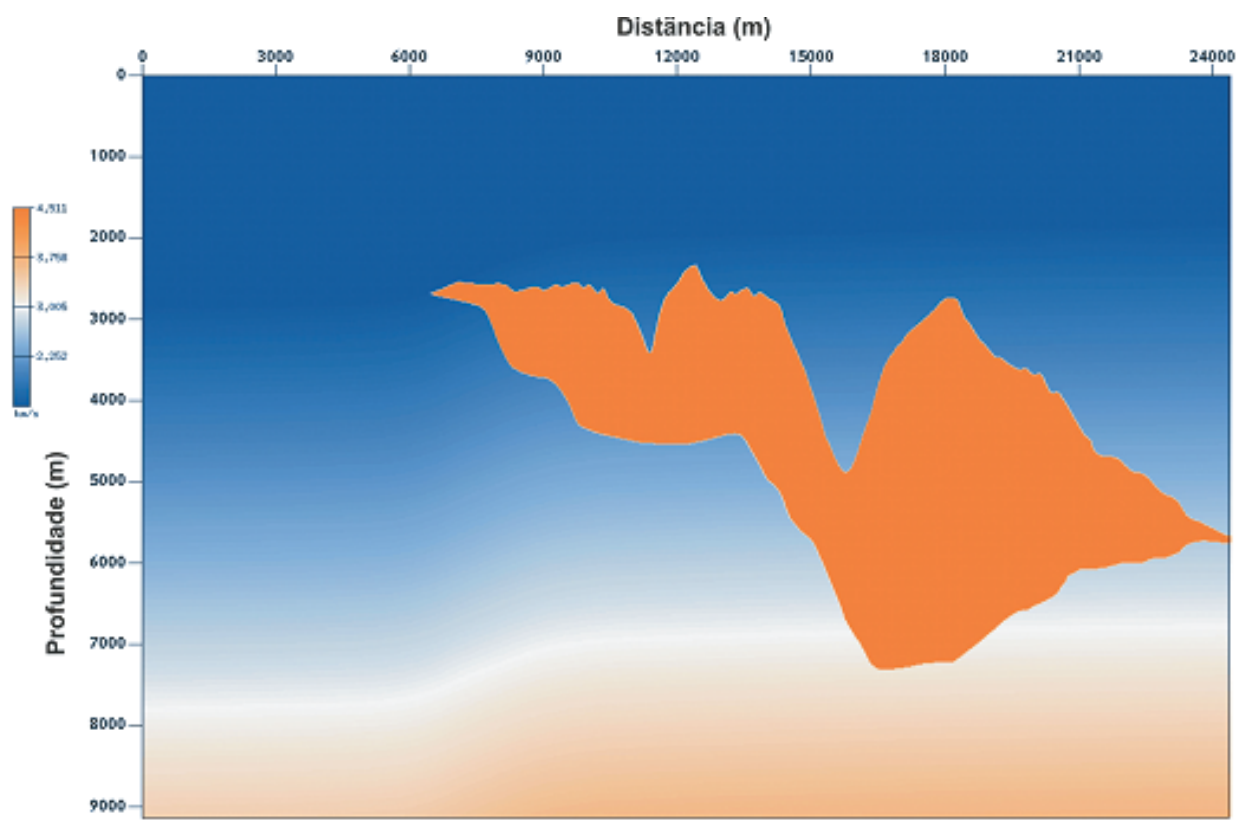

Figura 8 - Campo de velocidades do modelo SIGSBEE2A.

rador Split-Step (Figura 10) apresenta o domo de sal e as interfaces acima dele perfeitamente reconstituídos. Este método, como é aplicado recursivamente, não conseguiu nesses dados, devido ao forte contraste lateral de velocidade introduzido pelo corpo de sal, imagear corretamente os refletores abaixo do mesmo, bem como não apresenta os pontos difratores completamente colapsados.

Também a seção migrada com o método SS-S de tiro comum (Aldunate, 2002), Figura 11, mostra os refletores acima do domo de sal, as falhas e os pontos de difração, na parte esquerda do modelo até $7315,2 \mathrm{~m}$, bem imageados. Novamente, a reconstrução 


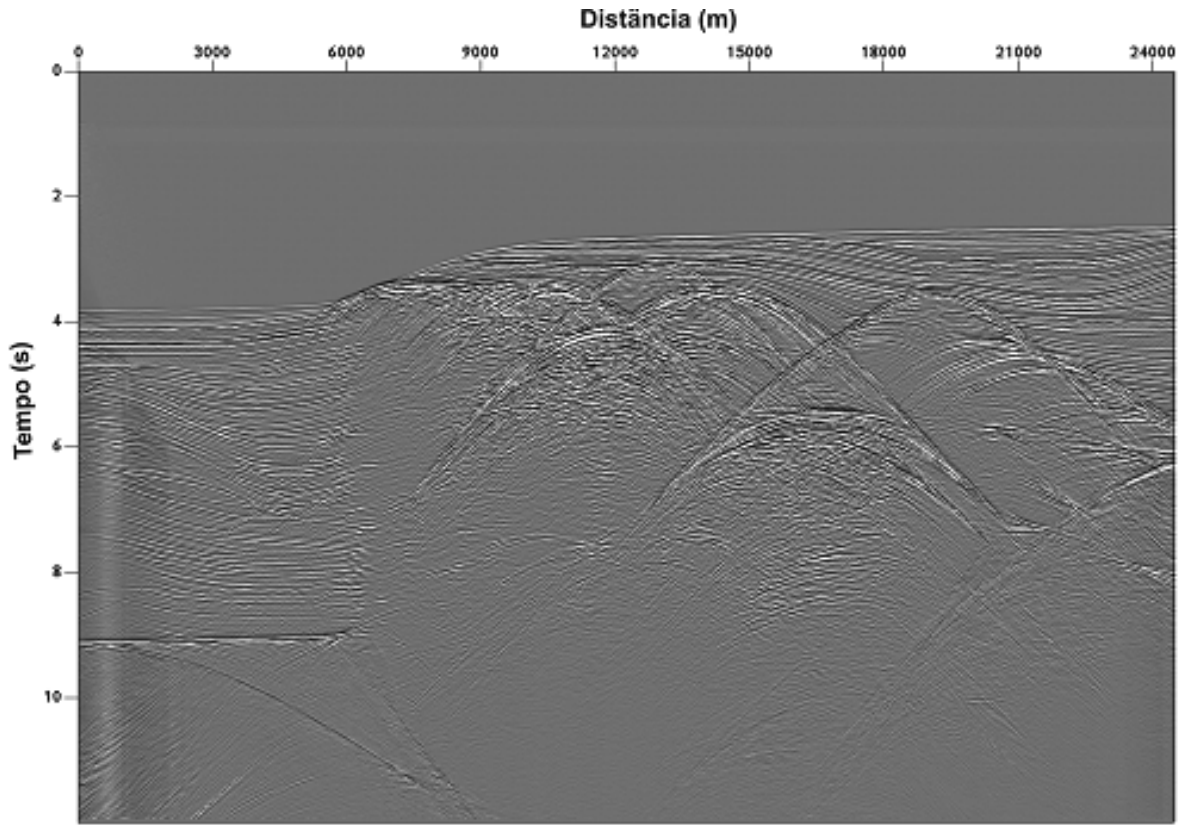

Figura 9 - Seção de afastamento nulo dos dados do modelo SIGSBEE2A.

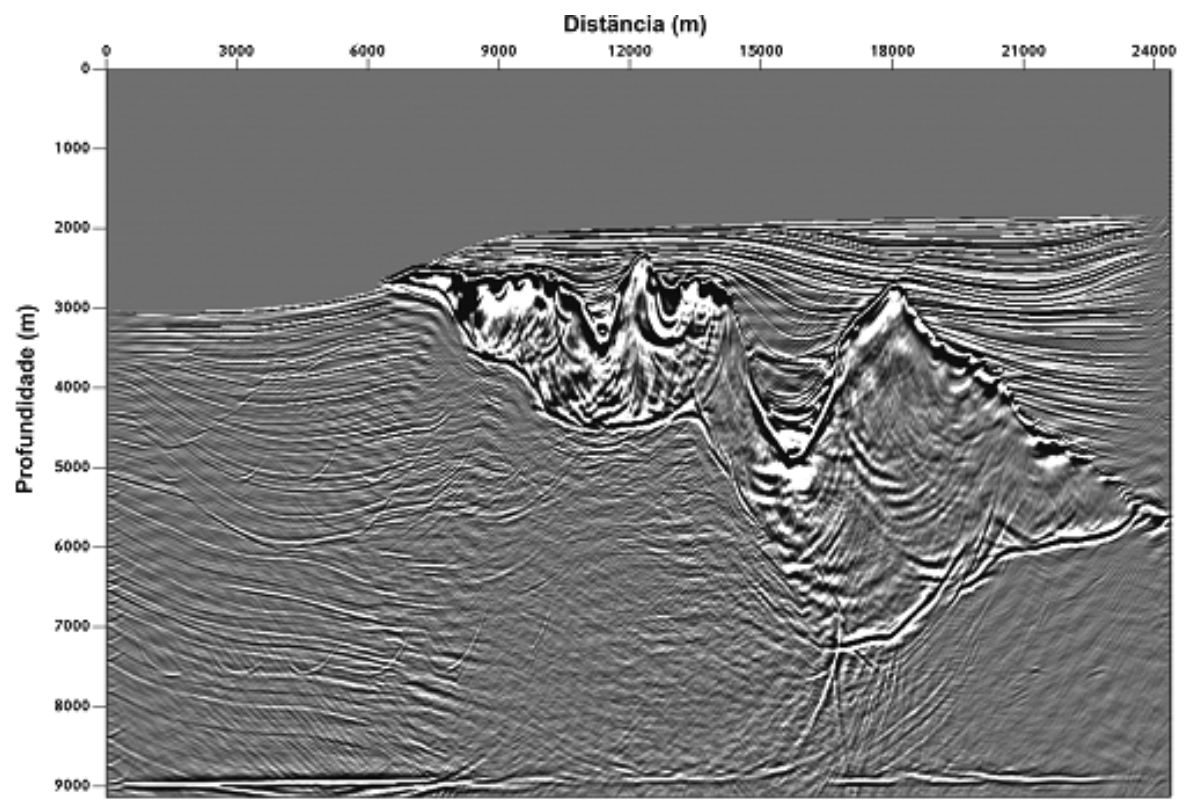

Figura 10 - Resultado da migração de fontes de ondas planas com operador Split-Step, do modelo SIGSBEE2A, obtido somando-se 81 seções de $p$ constante.

das feições abaixo do corpo de sal não foi conseguida, assim como no método de fontes de ondas planas com operador SplitStep.

Já o resultado da migração com o método PSPI-SS de tiro comum (Aldunate \& Pestana, 2004), Figura 12, apresenta um ótimo resultado, pois conseguiu imagear bem o domo de sal e os refletores acima dele. Os principais refletores e também as falhas abaixo do corpo de sal foram reconstituídos com sucesso. Os pontos difratores também foram completamente colapsados. Este resultado foi obtido usando-se apenas 3 velocidades de referência. 


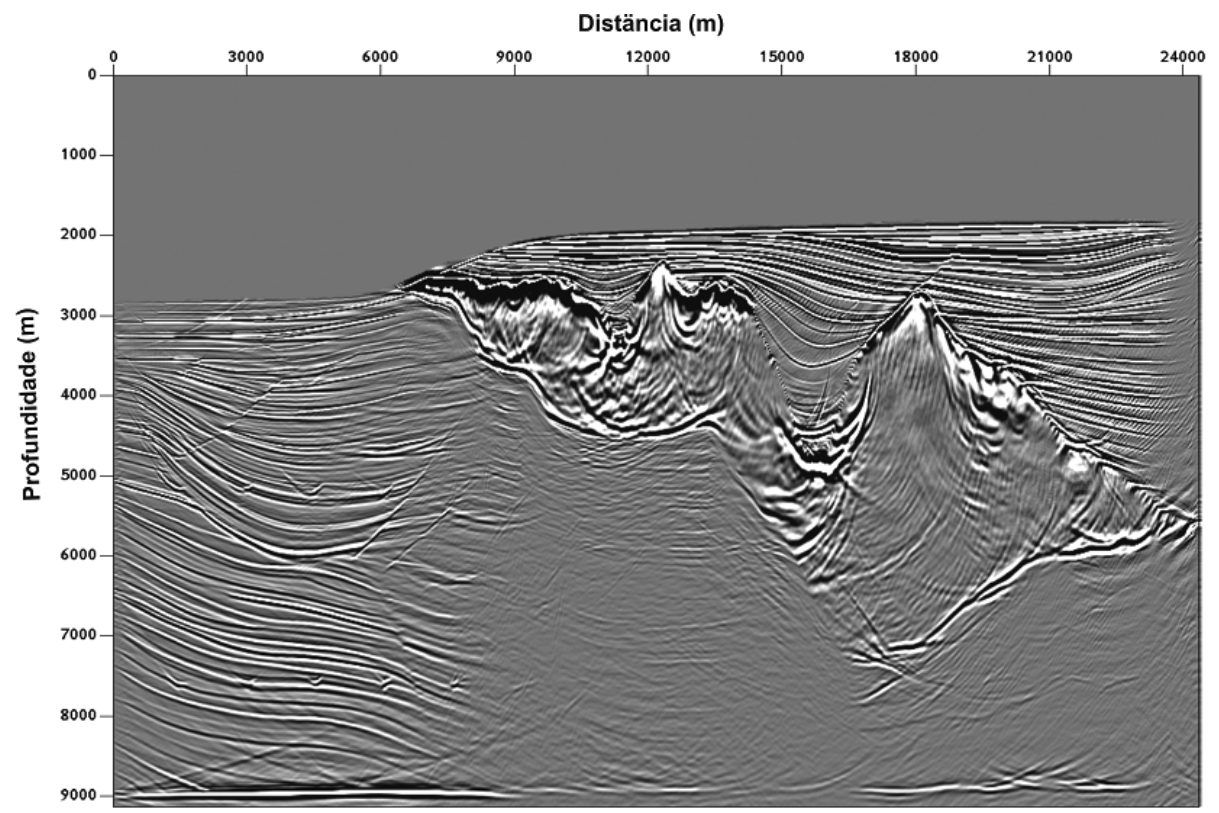

Figura 11 - Resultado da migração SS-S de tiro comum do modelo SIGSBEE2A.

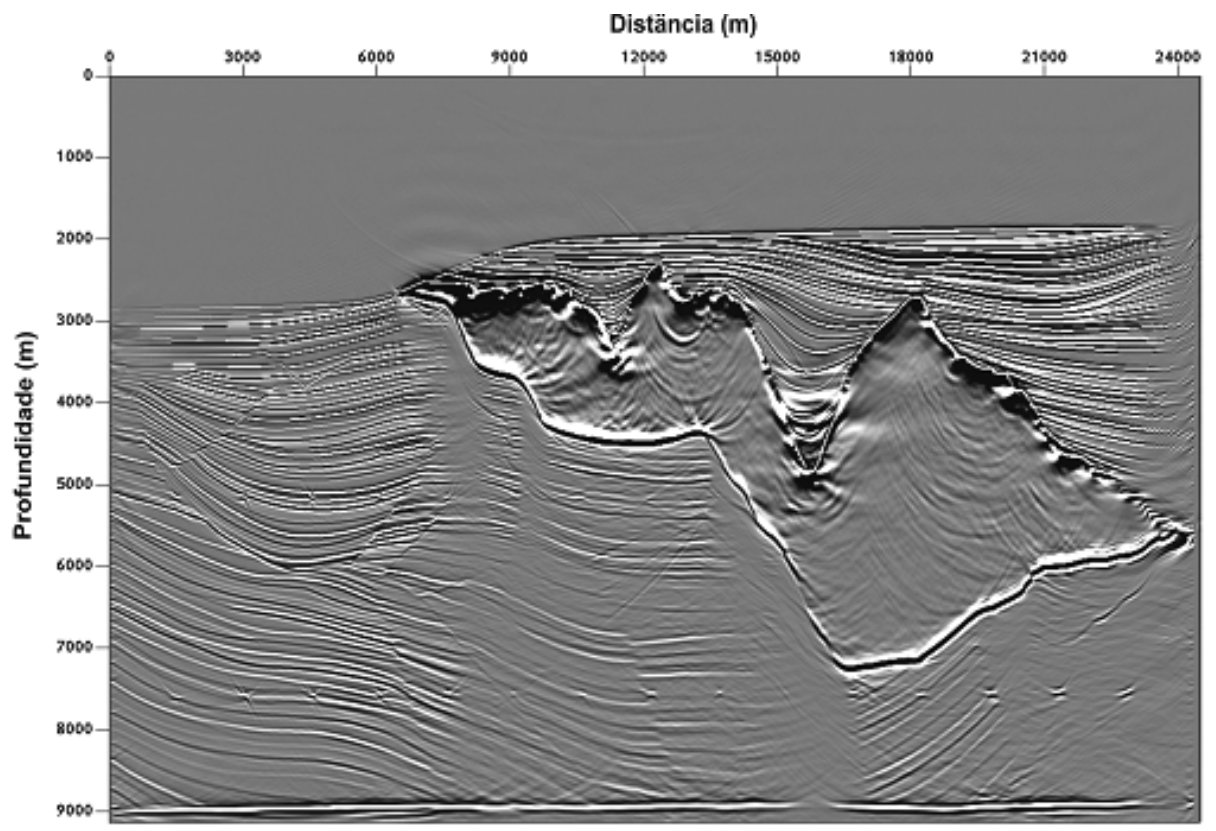

Figura 12 - Resultado da migração PSPI-SS de tiro comum do modelo SIGSBEE2A.

0 resultado da migração PSPI-SS de tiro comum (Figura 12) apresenta uma imagem migrada melhor do que a seção migrada com o método de fontes de ondas planas com operador SplitStep. Isto se deu em decorrência da forma como as velocidades de migração de referência são obtidas nos métodos PSPI-SS e SS-S de tiro comum. Como a migração é realizada em tiro co- mum, então as velocidades de referência são determinadas dentro da região relativa ao tiro e os seus respectivos receptores. E assim, para cada tiro, as velocidades de referência são diferentes. Desta forma, os métodos PSPI-SS e SS-S de tiro comum são implementados usando-se velocidades mais próximas das velocidades verdadeiras do modelo. No método de ondas planas 
com o operador Split-Step, a velocidade de referência é calculada considerando-se todo o campo de velocidades. Portanto, como podemos verificar, o resultado da migração de ondas planas com o operador Split-Step não apresenta a mesma qualidade que a migração SS-S de tiro comum, devido principalmente ao grande contraste lateral de velocidade introduzido pelo corpo de sal.

\section{Tempo de Processamento}

Os tempos de processamento das migrações apresentadas foram computados em uma máquina Pentium IV, com 2,4 GHz e 2 Gb de memória RAM. 0 tempo total de migração do modelo SEG-EAGE dos métodos de fontes de ondas planas com operador Split-Step foi de 2,5 horas, o tempo de processamento do método SS-S de tiro comum foi de 11,2 horas e o tempo de processamento do método PSPI-SS de tiro comum, para três velocidades de referência, foi de 18,6 horas. Já o tempo total de processamento do modelo SIGSBEE2A foi de 38 horas para o método de migração de fontes de ondas planas com Split-Step, de 63 horas para 0 método SS-S de tiro comum e de 96 horas para o método PSPISS de tiro comum também usando três velocidades de referência por passo de extrapolação.

\section{CONCLUSÕES}

Os resultados apresentados pelas migrações pré-empilhamento em profundidade de dados de fontes de ondas planas, métodos baseados na solução da equação da onda, de uma forma geral produziram seções migradas de boa qualidade atestando a capacidade desse método em imagear estruturas complexas com fortes variações laterais de velocidades.

Os resultados obtidos com essa nova metodologia de migração de ondas planas nos dois conjuntos de dados testados, conseguiram reconstituir os respectivos modelos em profundidade com bastante sucesso.

Os modelos SEG-EAGE e SIGSBEE2A são baseados em situações geológicas reais e representam estruturas geológicas complexas, associadas a fortes mergulhos e altos contrastes de velocidade. 0 conhecimento dos campos de velocidades destes modelos, bem como a posição correta dos refletores, permitiram avaliar melhor o desempenho dessa nova técnica de migração usando ondas planas.

No modelo SEG-EAGE os resultados obtidos pelos novos métodos apresentados neste artigo, ou seja, a migração de dados de fontes de ondas planas, com operador Split-Step, e o método híbrido foram comparáveis ao resultado da migração SS-S de tiro comum (Aldunate, 2002). Podemos, ainda, creditar o melhor re- sultado fornecido pelo procedimento usando o operador SplitStep, tanto para a extrapolação da fonte de onda plana, bem como os dados simulados em ondas planas, ao fato que à medida que o campo da onda plana, gerado na fonte, se propaga no modelo, tem sua direção alterada e também devido às correções laterais de velocidade aplicadas aos campos de ondas, durante o processo de extrapolação pelo operador Split-Step.

0 resultado do modelo SIGSBEE2A, apresentado pelo método Split-Step com dados de fontes de ondas planas, já não apresentou a mesma qualidade alcançada com os dados SEG-EAGE, pois os espalhadores não foram completamente colapsados e a região abaixo do domo de sal não apresenta os refletores bem focalizados. Isto se deve ao fato que neste método ao extrapolar o campo de ondas planas por todo o modelo utiliza-se apenas uma única velocidade de referência, em cada profundidade, e a mesma foi obtida tomando-se toda a extensão do modelo. Por sua vez, os resultados apresentados pelos métodos SS-S e PSPI-SS de tiro comum (Aldunate (2002); Aldunate \& Pestana (2004)) conseguiram imagens de boa qualidade, especialmente 0 resultado apresentado pelo método PSPI-SS, que mostra uma boa focalização dos refletores abaixo do corpo de sal e também todos os espalhadores completamente colapsados.

Comparando-se os tempos de processamentos totais, do modelo SEG-EAGE, dos métodos de dados de fontes de ondas planas com operador Split-Step com os métodos SS-S e PSPI-SS de tiro comum constatamos que: o método de fontes de ondas planas, com operador Split-Step, foi 4,5 vezes mais rápido do que o SS-S de tiro comum e 7,5 vezes mais rápido do que PSPI-SS. Mostrando assim uma melhora em termos computacional dos novos métodos apresentados neste trabalho. Ou seja, os métodos de migração de fontes de ondas planas, como operador SplitStep, forneceram resultados semelhantes aos alcançados com a migração SS-S de tiro comum a um menor custo computacional.

\section{AGRADECIMENTOS}

Os autores agradecem ao CTPETRO/CNPq-FINEP pelo apoio à Rede Cooperativa de Pesquisa em Risco Exploratório, ANP (Agencia Nacional de Petróleo), PETROBRAS e ao CPGG/UFBA pelo apoio no desenvolvimento deste trabalho. Reynam Pestana também agradece ao CNPq pelo suporte, através de bolsa de pesquisa, processo 300.312/88-1 (RM).

\section{REFERÊNCIAS}

ALDUNATE GC. 2002. Migração Pré-empilhamento Usando Operadores de Extrapolação tipo "split-step", Dissertação de Mestrado, Universidade Federal da Bahia. 
ALDUNATE GC \& PESTANA RC. 2004. Método de migração préempilhamento em profundidade baseado na equação acústica da onda. Workshop da Rede Cooperativa de Pesquisa em Risco Exploratório.

DUQUET B, LAILLY P \& EHINGER A. 2001. 3D Plane wave migration of streamer data, 71st Ann. Mgt., Soc. Expl. Geophysics.

FREIRE RML. 1988. Migração por mudança de fase em duas etapas, Tese de Doutorado, Universidade Federal da Bahia.

FARIA EL \& STOFFA PL. 1994. Traveltime computation in Transversely Isotropic media, Geophysics, 59: 272-281.

GLOGOVSKY V, LANDA E \& PAFFENHOLZ J. 2002. Integrated approach to subsalt depth imaging: synthetic case study, The Leading Edge, 21(12): 1217-1223.

JING X, FINN CJ, DICKEN TA \& WILLEN DE. 2000. Encoding multiple shot gathers in prestack migration, In: 70th Annual International Meeting SEG, Expanded Abstract.

LIU F, STOLT RH, HANSON DW \& DAY ES. 2002. Plane wave source, In: 72nd Annual International Meeting, Utah, USA, SEG.
LOGRADO JCG. 2002. Migração 2-D pré-empilhamento em profundidade utilizando técnicas de correção de fase em duas etapas, Dissertação de Mestrado, Universidade Federal da Bahia.

MOREIRA NETO CA. 2004. Migração pré-empilhamento em profundidade utilizando ondas planas, Tese de Doutorado, Universidade Federal da Bahia, Salvador, Bahia.

PESTANA RC \& STOFFA PL. 2001. Plane wave prestack time migration, Journal of Seismic Exploration, 9: 211-222.

PESTANA RC, STOFFA PL \& SANTOS JRSB. 2000. Plane wave prestack time migration, In: SEG 70th Annual Meeting, pp. 810-813.

SANTOS JRSB. 2000. Atenuação de múltiplas do fundo do mar e migração pré-empilhamento no domínio das ondas planas, Dissertação de Mestrado, Universidade Federal da Bahia.

STOFFA PL, FOKKEMA JT, FREIRE RML \& KESSINGER WP. 1990. SplitStep Fourier migration, Geophysics, 55(4): 410-421.

ZHANG Y, SUN J, NOTFORS C, GRAY S, CHERNIS L \& YOUNG J. 2003. Delayed shot 3D prestack depth migration, 65th EAGE Conference \& Exhibition, Extended Abstracts.

\section{NOTAS SOBRE OS AUTORES}

Celso Alves Moreira Neto é bacharel em Física pela UFMG (1999) e Doutor em Geofísica pela UFBA, em 2004. Atualmente é professor substituto do Instituto de Física da UFBA. Tem como principal interesse de pesquisa a migração de dados sísmicos.

Gary C. Aldunate é graduado em Física (UATF-B0/1997), mestre em Geofísica de Exploração de Petróleo (UFBA/2002) e atualmente é aluno do Curso de Doutorado em Geofísica da Universidade Federal da Bahia. As suas principais áreas de interesse são modelagem e processamento de dados sísmicos, com ênfase em migração de dados sísmicos.

Reynam C. Pestana é graduado em Física (UFBA/1983) e doutor em Geofísica pela UFBA, 1988. Pós-doutorado em Geofísica no Instituto de Geofísica da Universidade de Karlsruhe (Alemanha), 1989-1991, e de 1998-1999 no Instituto de Geofísica da Universidade do Texas em Austin. De 1988 até o presente é pesquisador do CPGG/UFBA, atuando no Grupo de Geofísica de Exploração de Petróleo. Desde 1992 é Professor Adjunto do Departamento de Geofísica Nuclear do Instituto de Física e da Pós-graduação em Geofísica da UFBA. Tem atuado no desenvolvimento de métodos e algoritmos de processamento e imageamento sísmicos. Mais recentemente, suas pesquisas envolvem técnicas de migração de dados sísmicos decompostos em ondas planas. É membro da SBGf e da SEG. 